\title{
Disordering to Order: de Vries Behavior from a Landau Theory for Smectic Phases
}

\author{
Karl Saunders, ${ }^{1, *}$ Daniel Hernandez, ${ }^{1}$ Staci Pearson, ${ }^{1}$ and John Toner ${ }^{2}$ \\ ${ }^{1}$ Department of Physics, California Polytechnic State University, San Luis Obispo, California 93407, USA \\ ${ }^{2}$ Department of Physics and Institute of Theoretical Science, University of Oregon, Eugene, Oregon 97403, USA
}

(Received 19 December 2006; published 8 May 2007)

\begin{abstract}
We show that Landau theory for the isotropic $(I)$, nematic $(N)$, smectic- $A$, and smectic- $C$ phases generically, but not ubiquitously, implies "de Vries" behavior: i.e., a continuous $A-C$ transition can occur with little layer contraction while the birefringence increases significantly once the system moves into the $C$ phase. Our theory shows that 1 st order $A-C$ transitions are also possible. These transitions can be de Vries-like, but in general need not be. Generally, de Vries behavior occurs in models with unusually small orientational order and is preceded by a first order $I-A$ transition. These results correspond well with experimental work to date.
\end{abstract}

DOI: 10.1103/PhysRevLett.98.197801

PACS numbers: 64.70.Md, 61.30.Gd, 61.30.Cz

Recently, an unusual new class of liquid crystals known as "de Vries smectic liquid crystals" [1] has drawn interest. They possess two defining features. First, there is little change with temperature $T$ of the layer spacing $d(T)$ upon entry to the $C$ phase, in contrast to the rapid geometrical contraction $d(T) \propto \cos \theta(T)$ expected if the molecules tilt by a strongly temperature dependent angle $\theta(T)$. Second, the birefringence increases significantly upon entering the $C$ phase from the $A$ phase. In fact, for de Vries materials with a 2 nd order $A-C$ transition, the birefringence is seen $[2,3]$ to decrease with decreasing temperature as the $A-C$ transition as approached, reaching a minimum at the $A-C$ transition. This is the first example known to us of decreasing order as a lower symmetry phase is approached. For de Vries materials with a 1st order transition, the birefringence increases moderately as the $A-C$ transition is approached and then jumps significantly at the transition [4]. Generally, de Vries smectic liquid crystals exhibit the phase sequence $I-A-C$, without a nematic phase. First order $A-C$ transitions are not always de Vries-like [4], contrary to some recent speculations.

In de Vries' "diffuse cone model" [5] of these materials, the molecules "pretilt" in the $A$ phase as the $A-C$ transition is approached, but in azimuthally random directions (hence reducing orientational order), so that there is no long range order in the tilting. Upon entering the $C$ phase, the molecules azimuthally order (hence increasing orientational order) without the significant layer contraction that occurs in conventional smectics whose molecules tilt at the $A-C$ transition.

In this Letter, we show that in a complete, nonchiral Landau mean field theory for the isotropic $(I)$, nematic $(N)$, $A$ and $C$ phases, in which all three order parameters (orientational, layering, azimuthal tilt) and the layer spacing are coupled, de Vries behavior occurs in a finite fraction of parameter space, while other regions exhibit conventional behavior. The mean field phase diagram for our model is shown in Fig. 1. Here, $t_{s}$ and $t_{n}$ are Landau theory parameters that control layering and orientational order, respec- tively. We find that two main features are necessary for de Vries behavior - an unusually weak coupling between layering and orientational order and a virtually temperature independent $t_{n}$. This latter feature would correspond to almost perfect excluded volume short range repulsive molecular interactions. Our theory also predicts that materials exhibiting de Vries behavior will almost always follow the phase sequence $I-A-C$. For 2 nd order $A-C$ transitions, we show that systems with an athermal $t_{n}$ will exhibit the unusual feature of a decreasing birefringence as the transition is approached from the $A$ phase. In fact, we predict

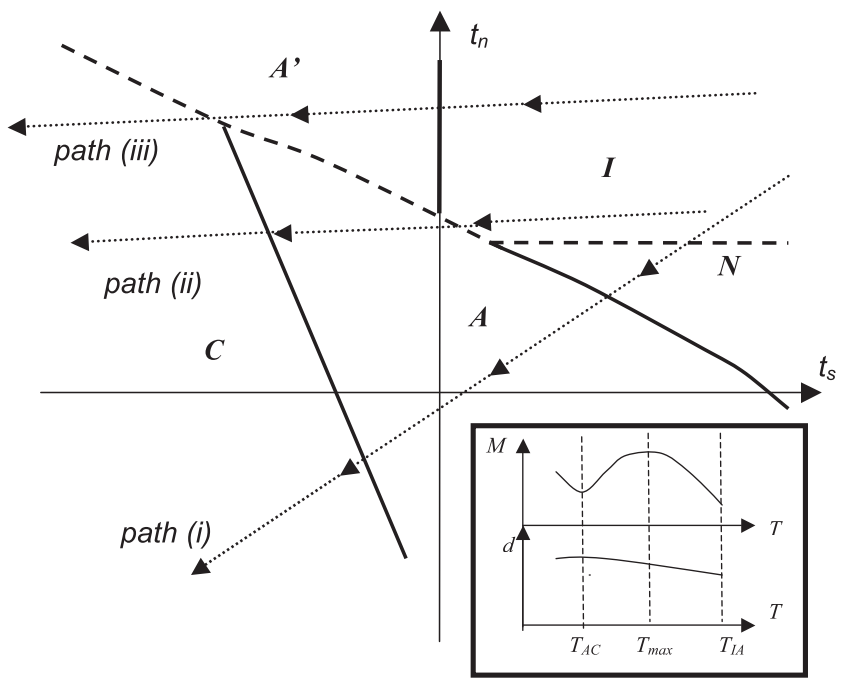

FIG. 1. The phase diagram in $t_{s}-t_{n}$ space for the $I, N, A$, and $C$ phases. 1 st and 2 nd order phase boundaries are shown as dashed and solid lines, respectively. Three decreasing temperature paths from the $I$ to $C$ phase are shown. Path (i) corresponds to a conventional material that does not exhibit de Vries behavior. Path (ii) corresponds to a material exhibiting de Vries behavior and a 2 nd order $A-C$ transition. Path (iii) leads to a 1 st order $A-C$ transition with de Vries behavior. The inset shows a possible temperature dependence of birefringence $(M)$ and layer spacing (d) for path (ii). 
that in the $A$ phase, some systems will exhibit a birefringence that has a nonmonotonic dependence on temperature, as shown in the inset of Fig. 1 [6]. Lastly, we also show that 1 st order $A-C$ transitions can (but generally need not) be de Vries-like.

Another result of our theory, unrelated to de Vries behavior, is that this phase diagram predicts two distinct smectic- $A$ phases of identical symmetry, denoted $A$ and $A^{\prime}$, separated by a first order phase transition. While 1 st order transitions between two smectic- $A$ phases due to competition between two different layer spacings [7] have been predicted, our result shows that even without such competition, $A-A$ transitions occur quite naturally and should be far more common than was previously thought.

Any experiment in which temperature is varied at fixed concentration traces a path through this phase diagram. As usual in Landau theory, we assume throughout this Letter that $t_{s}$ and $t_{n}$ are monotonically increasing functions of temperature; hence, as temperature is lowered, one moves monotonically from upper right to lower left in Fig. 1. Three qualitatively distinct paths of this type are shown. Path (i) is a typical path for a material that does not display de Vries behavior; along it, $t_{s}$ and $t_{n}$ both depend strongly on temperature. Paths (ii) and (iii) correspond to de Vries behavior with 2nd and 1st order $A-C$ and $A^{\prime}-C$ transitions, respectively. Both paths have strongly varying $t_{s}$ and weakly varying $t_{n}$; i.e, $t_{n}$ is virtually athermal. This would be the case if the $I-N$ transition is driven by a steric mechanism for which $t_{n}$ depends strongly on concentration and weakly on $T$. We find that de Vries behavior occurs in this case, for sufficiently weak coupling between layering and orientational orders. It should also be pointed out that a path above (iii), away from the $A-A^{\prime}-C$ critical end point (CEP), would exhibit a 1 st order $A^{\prime}-C$ transition without de Vries behavior.

This restriction to nearly horizontal paths implies that de Vries systems should very rarely exhibit an $N$ phase between the $A$ and $C$ phases, since to cross the $I-N$ boundary, a nearly horizontal path in Fig. 1 would have to be "fine tuned" to start very close to the boundary. The most likely paths to see de Vries behavior are those like (ii) or (iii), showing phase sequence $I-A-C$ or $I-A^{\prime}-C$, in good agreement with experimental work to date.

In this Letter, we focus on path (ii) and briefly discuss path (iii) at the end. The inset in Fig. 1 shows predictions for the layer spacing $d(T)$ and birefringence $M(T)$ as $T$ is varied along path (ii). The increase of the layer spacing in the $A$ phase as the $A-C$ transition is approached, though contrary to the de Vries picture of "pre- tilting" in the $A$ phase, is seen experimentally [2]. In the $A$-phase, our Landau theory predicts

$$
M_{A}(T)=M_{\max }-M_{2}\left[\frac{t_{s}(T)-t_{s}\left(T_{\max }\right)}{t_{s}\left(T_{\max }\right)}\right]^{2},
$$

where $M_{\max }, M_{2}$, and $T_{\max }$ are positive constants. We have shown that if $t_{n}$ is completely athermal, $T_{A C}<T_{\max }$, where
$T_{A C}$ is the $A-C$ transition temperature, so that in systems that follow a path like (ii), $M_{A}(T)$ will decrease as the $A-C$ transition is approached. A subset of such systems will have $T_{\max }<T_{I A}$, where $T_{I A}$ is the $I-A$ transition temperature, so that $M_{A}$ has a maximum within the $A$ phase, as shown in the inset in Fig. 1. If $t_{s}$ is linear in $T$ in the $A$-phase (as expected for small $T_{I A}-T_{A C}$ ), then $M_{A}(T)$ will be perfectly parabolic in $T$.

In the $C$ phase near the $A-C$ transition, the critical temperature dependences of $M$ and the tilt angle $\theta(T)$ predicted by our Landau theory are as follows: $M$ linear in $T$, and $\theta \propto\left(T_{A C}-T\right)^{1 / 2}$. When fluctuation effects are included, we expect [8] $\theta \propto\left(T_{A C}-T\right)^{\beta}$, where $\beta \approx 0.35$ is the order parameter critical exponent for the $3 \mathrm{D} X Y$ model. The layer spacing scales with temperature in our Landau theory according to

$$
d=d_{0}+a\left(T_{A C}-T\right)+b\left(T_{A C}-T\right)^{3},
$$

where $d_{0}$ is the value of the layer spacing at the $A-C$ transition and $a$ and $b$ are constants that depend on the Landau theory parameters . Clearly, if $a$ is sufficiently small, which we find is the case in the $C$ phase for sufficiently weak coupling between layering and orientational order, the layer spacing shows very little variation with temperature near $T_{A C}$. Significantly, we find that in cases like path (ii), the criterion for de Vries behavior of $d(T)$ differs from that for $M(T)$. Hence, we predict that some systems will exhibit de Vries behavior of the birefringence, but not de Vries behavior of the layer spacing.

How do fluctuations affect our mean field Landau theory? While our theory predicts that the $I-A^{\prime}$ transition is continuous, it is known [9] that fluctuations always drive the $I-A^{\prime}$ transition first order, albeit only weakly so if fluctuations are small. Fluctuation effects will also shift the positions of all of the transitions we have found. We expect, however, that the topology and essential geometry of the phase diagram Fig. 1 should occur in real systems. The only qualitative difference we expect is that the $I-A-A^{\prime}$ critical end point (CEP) predicted by Landau theory will be replaced by an $I-A-A^{\prime}$ triple point.

In other regions of parameter space, our model has an $N-A-C$ point; we will discuss this elsewhere [10].

In summary, de Vries behavior emerges quite naturally from our Landau theory. Equally importantly, conventional behavior (for both 1st and 2nd order transitions) also generically occurs for different Landau parameters. Thus, the model can accommodate all observed behaviors in all systems, and also predicts new behaviors not yet seen experimentally, like the first order $A-A^{\prime}$ transition.

We will now briefly describe the formulation and analysis of our theory. A Landau theory for all four phases $(I, N$, $A, C)$ must include order parameters for three types of order: uniaxial orientational order, tilt (azimuthal) order, and layering order. The first two are embodied in the usual second rank tensor orientational order parameter $\mathcal{Q}$. The complex layering order parameter $\psi$ is defined via the 
density $\rho=\rho_{0}+\operatorname{Re}\left(\psi e^{i \mathbf{q} \cdot \mathbf{r}}\right)$ with $\rho_{0}$ constant and $\mathbf{q}$ the layering wave vector. Taking both $\psi$ and $\mathcal{Q}$ to be spatially uniform implies the Landau free energy density $f=f_{Q}+$ $f_{\psi}+f_{c}$, with the orientational $\left(f_{Q}\right)$ [11], layering $\left(f_{\psi}\right)$, and coupling $\left(f_{c}\right)$ terms given by

$$
\begin{aligned}
f_{Q}= & \frac{t_{n} \operatorname{Tr}\left(Q^{2}\right)}{12}-\frac{w \operatorname{Tr}\left(Q^{3}\right)}{18}+\frac{u_{n}\left[\operatorname{Tr}\left(Q^{2}\right)\right]^{2}}{144}, \\
f_{\Psi}= & \frac{1}{2} t_{s}|\Psi|^{2}+\frac{1}{4} u_{s}|\Psi|^{4}+\frac{1}{2} K\left(q^{2}-q_{0}^{2}\right)^{2}|\Psi|^{2}, \\
f_{c}= & \frac{1}{2}\left[-g_{1}\left(q^{2}\right) q_{i} q_{j} Q_{i j}+g_{2} q_{i} q_{j} Q_{i k} Q_{j k}\right]|\Psi|^{2} \\
& +\frac{h}{4}\left(q_{i} q_{j} Q_{i j}\right)^{2}|\Psi|^{4},
\end{aligned}
$$

where $q_{i}$ is the component of the layering wave vector in the $i$ th direction, and the Einstein summation convention is implied. The constant $q_{0}$ is the magnitude that the layering wave vector would have in the absence of coupling between layering and orientational order. For weak coupling, $q \approx q_{0}$, and we Taylor expand $g_{1}\left(q^{2}\right) \approx g_{1_{0}}+g_{1}^{\prime}\left(q^{2}-\right.$ $\left.q_{0}^{2}\right)$, where $g_{1_{0}} \equiv g_{1}\left(q_{0}^{2}\right)$ and $\left.g_{1}^{\prime} \equiv \frac{d g_{1}}{d\left(q^{2}\right)}\right|_{q^{2}=q_{0}^{2}}$. The relatively large number of parameters in $f$ is inevitable given the fact that the theory incorporates three types of order. Furthermore, this theory allows for the possibility of conventional or de Vries behavior. However, we will show that in general, any of the five possible phases shown in Fig. 1 can be accessed by allowing, at most, two of the above parameters $\left(t_{n}\right.$ and $\left.t_{s}\right)$ to vary (with temperature). The presence or absence of the two defining features of de Vries behavior, small change in layer spacing and decreasing birefringence, depend only on the size of $g_{1}^{\prime}$ and the athermal nature of $t_{n}$, respectively. The remaining fixed positive parameters are required to stabilize the phases [12-14].

Choosing the arbitrary direction of the layer normals to be $z$, we seek the configuration of $\psi, \mathcal{Q}$, and $\mathbf{q}=q \hat{\mathbf{z}}$ that minimizes $f$. The form of $\mathcal{Q}$ that does so [10] is given by

$$
\begin{aligned}
Q_{i j}= & (-S+\sqrt{3} \eta) e_{1 i} e_{1 j}+(-S-\sqrt{3} \eta) e_{2 i} e_{2 j} \\
& +(2 S) e_{3 i} e_{3 j},
\end{aligned}
$$

where $\hat{\mathbf{e}}_{\mathbf{3}}=\mathbf{c}+\sqrt{1-c^{2}} \hat{\mathbf{z}}$ is the average direction of the molecules' long axes (i.e., the director). Here, in either smectic phase, $\hat{\mathbf{z}}$ is normal to the layers; in the $N$ and $I$ phases, the direction of $\hat{\mathbf{z}}$ is arbitrary. The projection, $\mathbf{c}$, of the director onto the layers is the order parameter for the $C$ phase. The other two principal axes of $\mathcal{Q}$ are given by $\hat{\mathbf{e}}_{\mathbf{1}}=$ $\hat{\mathbf{z}} \times \hat{\mathbf{c}}$ and $\hat{\mathbf{e}}_{\mathbf{2}}=\sqrt{1-c^{2}} \hat{\mathbf{c}}-c \hat{\mathbf{z}}$. $S$ and $\eta$ are proportional to the birefringence and biaxiality of the system, respectively. The $A$ phase is untilted $(\mathbf{c}=\mathbf{0})$ and uniaxial $(\eta=$ $0)$, while the $C$ phase is tilted $(\mathbf{c} \neq \mathbf{0})$ and biaxial $(\eta \neq 0)$. It is convenient to make the change of variables $S=$ $M \cos (\alpha)$ and $\eta=M \sin (\alpha)$. In the $A$ phase, $M$ is proportional to the birefringence.
We next minimize the free energy $f$ over the variables $M, \alpha, c,|\Psi|$, and $q$. Four qualitatively different types of minima are possible, corresponding to the four different symmetry phases $(I, N, A, C)$. Specifically, the $I$ phase has $M=0 ; \Psi=0$; the $N$ phase has $\Psi=0, \mathbf{c}=\mathbf{0}$, and $\alpha=0$, but $M \neq 0$; the $A$ phase has $\Psi \neq 0$ and $M \neq 0$, but $\mathbf{c}=\mathbf{0}$ and $\alpha=0$; and the $C$ phase has all of the variables $M, \alpha, \mathbf{c}$, and $\Psi \neq 0$. We render minimization analytically tractable by assuming that the coupling term Eq. (5) is small and by treating it perturbatively. Standard phase transition analysis [15] leads to the phase diagram shown in Fig. 1. Equations for the loci of the phase boundaries are given in [16]. The minimization of our Landau free energy also leads to predictions for the temperature dependences of $M$ and $q$. We find, in the $A$-phase,

$$
\begin{gathered}
M_{A}=M_{0}\left(t_{n}\right)+\frac{q_{0}^{2} \Psi_{0}^{2}}{\gamma}\left[-3 g_{2} M_{0}\left(t_{n}\right)+\delta\right], \\
q_{A}^{2}=q_{0}^{2}+\frac{M_{0}\left(t_{n}\right)}{K}\left[-g_{2} M_{0}\left(t_{n}\right)+g_{1}^{\prime} q_{0}^{2}+\delta\right],
\end{gathered}
$$

where $\quad \gamma \equiv w M_{0}\left(t_{n}\right)-2 t_{n}>0 \quad$ and $\quad M_{0}\left(t_{n}\right)=(w+$ $\left.\sqrt{w^{2}-4 u_{n} t_{n}}\right) / 2 u_{n}$ is the "bare" value of $M$, i.e., its value in the absence of coupling. Likewise, $\Psi_{0}=\sqrt{-t_{s} / u_{s}}$ is the bare value of $\Psi$. For strongly $T$ dependent $t_{s}$ and athermal $t_{n}$, the quantity $\delta$ is most usefully expressed as

$$
\delta\left(t_{s}, t_{n}\right) \equiv \zeta\left(t_{s}-t_{s}^{A C}\right),
$$

where $\zeta=\left(2 h q_{0}^{2} M_{0}\right) / u_{s}$, and $t_{s}^{A C}=-\left(g_{1_{0}}-g_{2} M_{0}\right) / \zeta$ is the value of $t_{s}$ where $\delta$ vanishes and the 2nd order $A-C$ transition occurs. In the $A$ phase, $\delta>0$ and in the $C$ phase $\delta<0$. In the $C$ phase, we find

$$
\begin{gathered}
M_{C}=M_{0}\left(t_{n}\right)+\frac{q_{0}^{2} \Psi_{0}^{2}}{\gamma}\left(-3 g_{2} M_{0}\left(t_{n}\right)-\frac{g_{2}}{2 h q_{0}^{2} \Psi_{0}^{2}} \delta\right), \\
q_{C}^{2}=q_{A}^{2}\left(t_{s}^{A C}\right)+\frac{g_{1}^{\prime}}{2 K h \Psi_{0}^{2}} \delta,
\end{gathered}
$$

where a decrease in layer spacing requires $g_{1}^{\prime}<0$. Finally, in the $A^{\prime}$-phase, we find

$$
\begin{gathered}
M_{A}^{\prime}=\frac{q_{0}^{2} \Psi_{0}^{2} g_{1_{0}}}{t_{n}}, \\
q_{A}^{\prime 2}=q_{0}^{2}\left(1+\frac{2 g_{1_{0}}^{2} \Psi_{0}^{2}}{K t_{n}}\right) .
\end{gathered}
$$

These results imply de Vries behavior for both birefringence and layer spacing. For a nearly horizontal experimental locus like path (ii) through the $A$ phase, the $T$ dependence of $M$ (and hence birefringence) in Eq. (7) comes from the linear $t_{s}$ - dependence of each of $\Psi_{0}^{2}$ and $\delta$ in the correction due to the coupling of layering and orientational orders. From Eq. (7), we see that nonmonotonicity of $M_{A}$ is due to a competition between the layering 
order, $\Psi_{0}^{2}$ and the coupling $\delta$ which increase and decrease, respectively, as the $A-C$ transition is approached. This happens because, as the system moves deeper into the $A$ phase, the layering order increases, thereby augmenting the weak orientational order due to the coupling between the two. However, as the $A-C$ transition (where at the director tilts away from the layer normal) is approached, this coupling necessarily decreases and, hence, so does $M_{A}$, which means the birefringence decreases as the $A-C$ transition is approached. Once in the $C$ phase, the birefringence begins to increase with decreasing $t_{s}$. It is straightforward to show that if $g_{1_{0}}>4 g_{2} M_{0}$, then $M_{A}$ exhibits a maximum inside the $A$ phase, as shown in the inset in Fig. 1.

As the $A-C$ transition is approached within the $A$ phase, $q_{A}$ monotonically decreases and hence $d$ monotonically increases. This is typical of both conventional and de Vries smectics although, as discussed above, it is somewhat contrary to the diffuse cone picture. The $T$ dependence of the layer spacing at the transition depends crucially on the size of the parameter $g_{1}^{\prime}$. In systems where the coupling of the layering and orientational order depends weakly on layer spacing and $\left|g_{1}^{\prime}\right|$ is unusually small, the $T$-dependence of $q$ is almost flat. We have shown that if $g_{1}^{\prime}=0$, then the change in layer spacing scales like $\left(T_{A C}-\right.$ $T)^{3}$ and hence varies very weakly in the $C$ phase near the $A-C$ transition. Systems with larger values of $\left|g_{1}^{\prime}\right|$ will have conventional behavior of the layer spacing.

Since this de Vries behavior of $q$ has a different criterion than the de Vries behavior of $M$ (which relies on $t_{n}$ being athermal), it should be possible to find systems which exhibit de Vries behavior of the birefringence, but not de Vries behavior of the layer spacing.

For systems that approach the $C$ phase from the $A^{\prime}$ phase, along path (iii), the birefringence will increase monotonically before jumping substantially (on the order of $M_{0}$ ) at the transition. From Eq. (13), we see that the $T$ dependent piece of $q_{A}^{\prime}$ is second order in the coupling $g_{1_{0}}$ which we treat perturbatively in our analysis. Thus, this $T$ dependent piece is very small in the $A$ phase. Upon entry into the $C$ phase, the $T$ dependence of the layering spacing will be weak if $g_{1}^{\prime}$ is small. At the $A^{\prime}$ - $C$ transition, there will be a jump in $q$ but Eqs. (8), (11), and (13) can be used to show that this jump will be small when $g_{1}^{\prime}$ and $g_{2}$ are small. For such a system, a transition just above the CEP will exhibit an almost continuous change in tilt angle, a weakly varying layer spacing, a substantial jump in birefringence, and a latent heat. Systems entering the $C$ phase well above this CEP will exhibit jumps in all of the above quantities and thus not be de Vries-like.

The requirement of near $T$-independence of $t_{n}$ for de Vries behavior severely restricts the possible experimental loci in Fig. 1 that can display such behavior: namely, nearly horizontal ones. A significantly sloped path like (i) will not exhibit de Vries behavior. In this case, the growth of the bare (i.e., coupling-free) birefringence $M_{0}\left(t_{n}\right)$ as $T$ is lowered swamps the effects due to the coupling terms, and makes the behavior of both the birefringence and the layer spacing conventional. Thus, our model can accommodate either conventional behavior or de Vries behavior, if $t_{n}$ is thermal or athermal, respectively.

We acknowledge very helpful discussions with Yuri Panarin. K. S., D. H, S.P. were sponsored by the Department of the Navy, Office of Naval Research.

*Corresponding author: ksaunder@calpoly.edu

[1] For an excellent review of de Vries smectics, see J.P.F. Lagerwall and F. Giesselmann, Chem. Phys. Chem. 7, 20 (2006).

[2] J.P. F. Lagerwall, F. Giesselmann, and M.D. Radcliffe, Phys. Rev. E 66, 031703 (2002).

[3] Y. Panarin (private communication).

[4] N. Hayashi, A. Kocot, M. J. Linehan, A. Fukuda, J. K. Vij, G. Heppke, J. Naciri, S. Kawada, and S. Kondoh, Phys. Rev. E 74, 051706 (2006).

[5] A. de Vries, Mol. Cryst. Liq. Cryst. Lett. 41, 27 (1977); A. de Vries, A. Ekachai, and N. Spielberg, ibid. 49, 143 (1979).

[6] Unfortunately, published data on birefringence of these materials within the $A$ phase seems only to focus very near the $A-C$ transition, although such nonmonotonicity has been seen in DSi3-MR11 [3].

[7] P. Barois, J. Prost, and T. C. Lubensky, J. Phys. (Paris) 46, 391 (1985).

[8] G. Grinstein and R. A. Pelcovits, Phys. Rev. A 26, 2196 (1982).

[9] S. A. Brazovskii and S. G. Dmitriev, Zh. Eksp. Teor. Fiz. 69, 979 (1975) [Sov. Phys. JETP 42, 497 (1976)].

[10] K. Saunders and J. Toner (to be published).

[11] P. G. De Gennes and J. Prost, The Physics of Liquid Crystals (Oxford University Press, New York, 1995).

[12] It should be noted that for the system to exhibit an $N-A-C$ multicritical point, a term $\left(q_{i} q_{j} Q_{i j}\right)^{2}|\Psi|^{2}$ must be included.

[13] We have for simplicity dropped some terms (e.g., $\operatorname{Tr}\left(\mathcal{Q}^{2}\right)|\Psi|^{2}$ ) that are actually lower order in the presumed small fields $\Psi$ and $\mathcal{Q}$ than the terms we have kept. We have verified that keeping such terms with small, but nonzero, coefficients has no qualitative effect on our results. In contrast, dropping any one of the terms we have kept is unphysical.

[14] A free energy similar to ours was used by P. K. Mukherjee et al., J. Chem. Phys. 117, 7788 (2002) to investigate first order $I-A$ and $I-C$ transitions. However, they ignored the biaxiality of the orientational order parameter and incorrectly claimed that the $C$ phase is stabilized by the $g_{2}$ term. This last mistake led them to exclude the $h$ term, which is necessary to stabilize the $C$ phase.

[15] See, for example, P.M. Chaikin and T. C. Lubensky, Principles of Condensed Matter Physics (Cambridge University Press, Cambridge, 1995).

[16] See EPAPS Document No. E-PRLTAO-98-078718 for equations for the phase boundaries. For more information on EPAPS, see http://www.aip.org/pubservs/epaps.html. 